\title{
MEGA: Microlensing Exploration of the Galaxy and Andromeda
}

\author{
David R. Alves, Edward Baltz, Arlin Crotts, Alex Bergier, Patrick \\ Cseresnjes, and Alan Gersch \\ Columbia Astrophysics Laboratory, 550 W. 120th St., Mailcode 5247, \\ New York, NY 10027, USA
}

\begin{abstract}
Microlensing surveys have ruled out that the dark halos of the Milky Way and M31 are composed entirely of massive compact halo objects (MACHOs) for a wide range of MACHO masses. We have tried using the Subaru telescope to improve the limit on Moon-mass MACHOs, which is the lowest decade in MACHO mass that can be probed by microlensing. Unfortunately, only a half-night of Subaru data was obtained and this is not enough; at least 2 nights of data are required. The current limit is based on nearly 6 years of observations with smaller telescopes.
\end{abstract}

\section{Introduction}

Naively, the Galactic dark halo could be composed entirely of objects that are less massive than $1 \%$ of the Sun because these mass objects must be dark. However, other limits apply. For example, the dark halo cannot be made entirely of objects with masses of about $10^{-10} M_{\odot}$ otherwise the sky would be filled with comets (Carr \& Sakellariadou 1999). Microlensing surveys have shown that no more than $5 \%$ of the dark halo could be composed of objects with masses from $10^{-7}$ to $10^{-3} M_{\odot}$ (Alcock et al. 1998). In the allowed low-mass range MACHOs could be made of solid hydrogen (White 1996), or strange quarks (Banerjee et al. 2003), or they could be black holes (Green \& Liddle 1999).

\section{Microlensing with an 8-m Telescope}

The venue for pushing the microlensing limits to smaller masses is not the Magellanic Clouds, where Alcock et al. (1998) searched, but in a more distant galaxy like M31 (e.g., Baltz, Gyuk \& Crotts 2003). Here the loss of detectability that results from the finite size of the source does not become a problem until the lenses in the Galactic halo are less than $10^{-8} M_{\odot}$, i.e. Moon-mass. However, to acquire enough signal on faint source stars in M31 on sub-hourly timescales requires a large telescope. The MEGA survey was allocated 2 nights with Subaru for this experiment. Due to weather, only $1 / 2$ night of useful data was obtained. In addition, a significant fraction of the M31 signal in this dataset was also lost due to a readout problem with one CCD in the mosaic imager.

Figure 1 shows the number of microlensing events expected in each dataset for a $100 \%$ halo of MACHOs as a function of MACHO mass. The significance of 


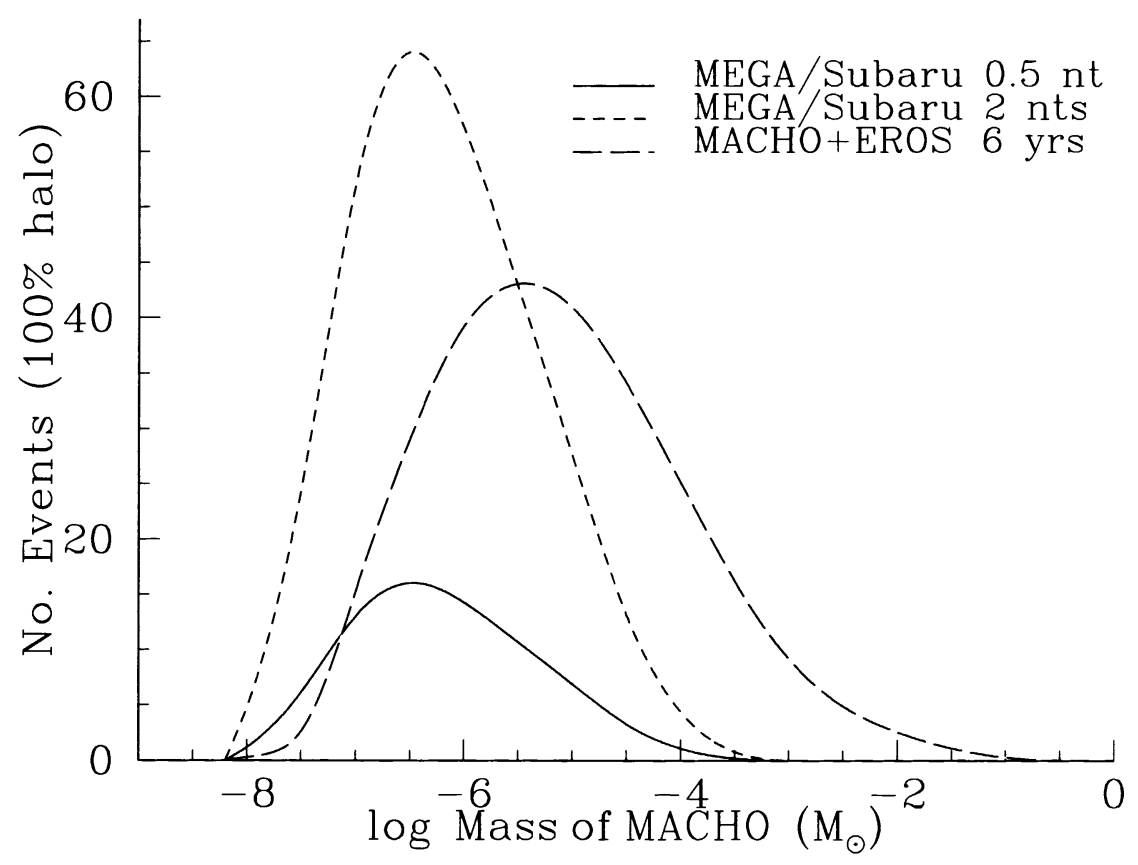

Figure 1. Predicted number of events for a $100 \%$ halo as a function of MACHO mass for different microlensing datasets. The long-dashed line shows the prediction from Alcock et al. (1998) for their 6-year dataset (M. Lehner, priv. comm.). MEGA's prediction for a half-night (solid line) and two nights (short-dash line) of Subaru data are overplotted.

the limit on the halo fraction is proportional to the square-root of the number of predicted events if no microlensing is detected. Although we predict slightly more low-mass events in the half-night dataset compared to Alcock et al. (1998), the actual number would be less because of the data lost to the malfunctioning CCD. In 2 nights an as yet unprobed decade in MACHO mass could be ruled out.

\section{References}

Alcock, C., et al. 1998 ApJ, 499, L9

Baltz, E., Gyuk G., \& Crotts A. 2003, ApJ, 582, 30

Banerjee, S. et al. 2003, MNRAS, 340, 284

Carr, B.J. \& Sakellariadou, M. 1999, ApJ, 516, 195

Green, A.M. \& Liddle, A.R. 1999 PRD, 60, 063509-1

White, R. S. 1996, Ap\&SS, 240, 75 